\title{
TRIM24 links a non-canonical histone signature to breast cancer
}

Wen-Wei Tsai ${ }^{1 *}$, Zhanxin Wang ${ }^{3 *}$, Teresa T. Yiu ${ }^{1,2}$, Kadir C. Akdemir $^{2,5}$, Weiya Xia $^{4}$, Stefan Winter ${ }^{6}$, Cheng-Yu Tsai ${ }^{7}$, Xiaobing Shi ${ }^{1,2}$, Dirk Schwarzer ${ }^{8}$, William Plunkett ${ }^{7}$, Bruce Aronow $^{9}$, Or Gozani ${ }^{10}$, Wolfgang Fischle ${ }^{6}$, Mien-Chie Hung ${ }^{4,11}$, Dinshaw J. Patel ${ }^{3} \&$ Michelle Craig Barton ${ }^{1,2}$

Recognition of modified histone species by distinct structural domains within 'reader' proteins plays a critical role in the regulation of gene expression. Readers that simultaneously recognize histones with multiple marks allow transduction of complex chromatin modification patterns into specific biological outcomes. Here we report that chromatin regulator tripartite motif-containing 24 (TRIM24) functions in humans as a reader of dual histone marks by means of tandem plant homeodomain (PHD) and bromodomain (Bromo) regions. The three-dimensional structure of the PHD-Bromo region of TRIM24 revealed a single functional unit for combinatorial recognition of unmodified H3K4 (that is, histone H3 unmodified at lysine 4, H3K4me0) and acetylated H3K23 (histone $\mathrm{H} 3$ acetylated at lysine 23, H3K23ac) within the same histone tail. TRIM24 binds chromatin and oestrogen receptor to activate oestrogen-dependent genes associated with cellular proliferation and tumour development. Aberrant expression of TRIM24 negatively correlates with survival of breast cancer patients. The PHD-Bromo of TRIM24 provides a structural rationale for chromatin activation through a non-canonical histone signature, establishing a new route by which chromatin readers may influence cancer pathogenesis.

Post-translational modifications of histones occur in combinations that must be faithfully translated by effector proteins, or histone readers ${ }^{1-4}$. The lexicon of histone modifications may be highly context-dependent, influenced by inductive signalling, cellular milieu and target gene status ${ }^{4}$. Misinterpretation or imbalance in this hierarchal arrangement has dire consequences for cellular homeostasis, leading to developmental problems, hereditary disease or tumour development ${ }^{5}$. Linked histone reader modules, such as tandem PHD finger and bromodomain, occur frequently in proteins that interact with histone, but little is known about their mechanisms of action. Combinatorial readout of histone posttranslational modifications (PTMs) may enhance binding between spatially separated histone marks, or even create communication links between domains or members of the complex ${ }^{3}$. Individually, proteins with bromodomains-for example, TAF1 and BDF1-associate with acetylated lysines with broad specificity ${ }^{6,7}$, while PHD-containing proteins are less predictable in their interactions ${ }^{1-4}$. The PHD fingers of BHC80 and AIRE interact with unmethylated $\mathrm{H} 3 \mathrm{~K} 4(\mathrm{H} 3 \mathrm{~K} 4 \mathrm{me} 0)^{8,9}$, while other previously reported PHD finger domains bind methylated proteins as modifiers of histones or as subunits of chromatinremodelling, co-activator or co-repressor complexes ${ }^{1-4,10-17}$.

PHD-finger proteins and their dysregulation are linked to a broad spectrum of human diseases, underscoring an essential role in homeostasis $^{5}$. Recently, aberrant localization of a JARID1A PHD fingerfusion protein was shown as directly causal in transformation and development of haematopoietic malignancy, which is a process requiring fusion protein recognition of $\mathrm{H} 3 \mathrm{~K} 4 \mathrm{me} 3$ via the JARID1A PHD finger ${ }^{18}$. Here, we present evidence that a multi-functional protein, TRIM24, which is an E3-ubiquitin ligase that targets p53 (ref. 19) and is broadly associated with chromatin silencing ${ }^{20}$, relies on PHD-Bromo to recognize specific, combinatorial histone modifications and activate oestrogen-dependent genes associated with cellular proliferation and tumour development. Genome-wide analysis of chromatin interactions shows oestrogen-dependent binding of TRIM24 and oestrogen receptor $\alpha(\mathrm{ER} \alpha)$ at sites that paradoxically exhibit oestrogen-activated loss of H3K4me2 and gain of histone acetylation. Importantly, aberrant overexpression of TRIM24 in breast cancer patients is frequent and directly correlated with poor survival.

\section{TRIM24 PHD-Bromo binds amino-terminal H3 tail}

TRIM24 belongs to the TRIM/RBCC protein family, characterized by a conserved, N-terminal tripartite motif-namely, a RING domain, B-box zinc-fingers, and a coiled-coil region-as well as variable carboxyterminal domains ${ }^{21,22}$. TRIM24 was originally identified as transcriptional intermediary factor (TIF) $1 \alpha$, a ligand-dependent, co-repressor of retinoic acid receptor that interacts with multiple nuclear receptors in vitro via an LXXLL motif ${ }^{23}$. In addition to its LXXLL motif and RING domain, TRIM24 has a C-terminal, PHD-Bromo (Fig. 1a), which probably recognizes histones or non-histone proteins with specific combinations of post-translational modifications.

Protein sequence alignment of the PHD fingers of TRIM24 and BHC80 with ING1, a PHD domain that recognizes H3K4me3 (refs 24,25), showed TRIM24 as highly similar to BHC80 with conservation of residues critical for $\mathrm{BHC} 80-\mathrm{H} 3 \mathrm{~K} 4 \mathrm{me} 0$ interactions $^{8}$

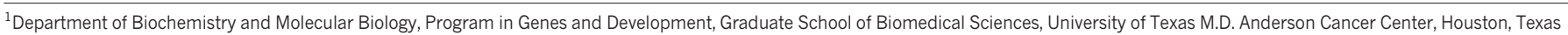
77030, USA. ${ }^{2}$ Centers for Cancer Epigenetics and Stem Cell and Developmental Biology, University of Texas M.D. Anderson Cancer Center, Houston, Texas 77030 , USA. ${ }^{3}$ Structural Biology Program,

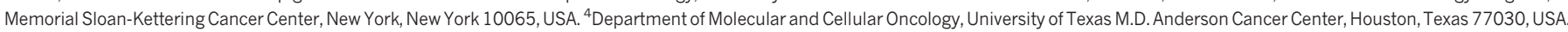

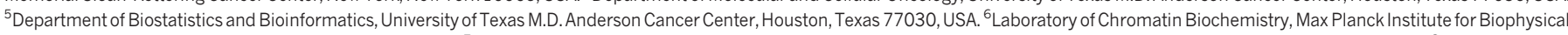

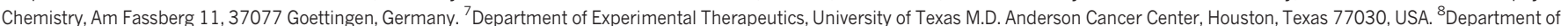

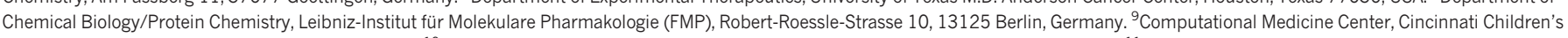

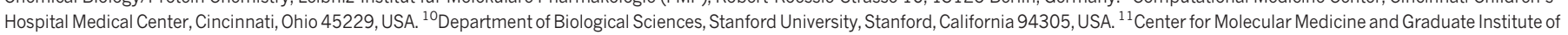
Cancer Biology, China Medical University and Hospital, Taichung 404, Taiwan.

*These authors contributed equally to this work. 



Figure 1 TRIM24 PHD finger interacts with unmethylated H3K4. a, Diagram of TRIM24 protein domains. b, Biotinylated peptide pulldowns: recombinant PHD fingers and histone peptides. c, GST-pulldowns: recombinant proteins and native histone proteins, $\mathrm{PHD}$-Bromo (PB). d, ITC titration: binding of TRIM24 PHD-Bromo with histone peptides.

(Supplementary Fig. 1a). Accordingly, we found that full-length TRIM24 interacts with histone proteins specifically through its PHD-Bromo (Supplementary Fig. 1b). Binding of the TRIM24 PHD-Bromo to histone peptide arrays occurs at unmodified H3 (residues 1-21), methylated H3K9 (H3K9me) and acetylated H3K9/K14 peptides, but not methylated H3K4 residues (Supplementary Fig. 1c). Similarly, TRIM24 PHD finger and PHD-Bromo bind unmodified histone $\mathrm{H} 3$ (residues 1-21) but not methylated H3K4, similar to BHC80 but unlike ING1, which preferentially binds to H3K4me peptides (Fig. $1 \mathrm{~b}$ and Supplementary Fig. 1d). Glutathione $S$-transferase (GST)-pulldown assays with native histones confirmed that TRIM24 PHD finger, bromodomain, PHD-Bromo and the BHC80 PHD fail to bind to native histone $\mathrm{H} 3$ with $\mathrm{K} 4$ trimethylation (H3K4me3) but tolerate H3K9me2 modification (Fig. 1c and Supplementary Fig. 1e). Isothermal titration calorimetry (ITC)-based binding assays established that the PHD-Bromo binds unmodified H3(1-15)K4 with a dissociation constant, $K_{\mathrm{D}}$, of $8.6 \mu \mathrm{M}$, while methylation of $\mathrm{H} 3 \mathrm{~K} 4$ greatly decreases binding affinity of TRIM 24 and $\mathrm{H} 3$ peptides (Fig. 1d and Supplementary Table 2). These results suggest that TRIM24 PHD-Bromo interacts with the N-terminal tail of histone
$\mathrm{H} 3$, but that specific PTMs, for example, methylation of H3K4, interfere with this interaction.

\section{Structural basis of $\mathrm{H} 3$ readout by TRIM24}

We have determined the three-dimensional crystal structure of the PHD-linker-Bromo segment (residues 824-1006) of human TRIM24 in free and histone peptide bound states. The overall structure of TRIM24 PHD-Bromo in the free state demonstrates that PHD and bromodomain interact extensively and form an integrated structural unit $\left(747 \AA^{2}\right.$ of contact surface), connected by a long linker and stabilized by a network of hydrogen bonding and hydrophobic interactions (Fig. 2a, Supplementary Fig. 2, Supplementary Table 1). The TRIM24 PHD finger residues 824-871 adopt the typical PHD finger 'cross-braced' topology stabilized by a pair of coordinated zinc ions, which together with residues 872-884 from the linker region form an extended TRIM24 PHD domain. The TRIM24 bromodomain adopts the typical left-handed four-helical bundle characteristic of other members of this family.

The 2.0 A co-crystal structure of TRIM24 PHD-Bromo and unmodified H3(1-10)K4 peptide (Supplementary Table 1 and Supplementary Fig. 3a) showed that the first nine residues of bound $\mathrm{H} 3$ peptide are positioned within a surface groove of the PHD finger (Fig. $2 \mathrm{~b}$ and Supplementary Fig. 3b). The R2 to Q5 segment of bound H3 peptide forms an anti-parallel $\beta$-sheet with the E837 to C840 segment of the PHD finger, while the T6 to K9 segment of bound $\mathrm{H} 3$ peptide contacts the N834 to G836 segment of the PHD finger. The side chain of R2 is hydrogen-bonded with the backbone carbonyl of C841. The side chain of C840 is positioned in-between the side chains of $\mathrm{R} 2$ and $\mathrm{K} 4$, with the C840W mutation losing its ability to bind unmodified H3K4 peptide $\left(K_{\mathrm{D}}>400 \mu \mathrm{M}\right.$, Supplementary Table 2 and Supplementary Fig. 4) .

The unmodified lysine ammonium group of $\mathrm{H} 3 \mathrm{~K} 4$ forms two direct hydrogen bonds with backbone carbonyl oxygens of N825 and E826 (Fig. 2b). In addition, the proximally positioned D827 forms a stabilizing salt bridge with the unmodified lysine, consistent with the observation of impaired binding between D827A mutant and unmodified H3K4 peptide $\left(K_{\mathrm{D}}=133 \mu \mathrm{M}\right.$, Supplementary Table 2). Methylation of H3K4 would create steric clashes with residues lining the binding pocket, disrupt the salt bridge interaction with D827, and impair hydrogen bonding with N825 and E826, thereby providing a structural explanation for the unmodified $\mathrm{H} 3 \mathrm{~K} 4$ preference of TRIM24 PHD-Bromo.

\section{TRIM24 bromodomain is H3K23ac-specific}

Both sequence and structure-based alignments indicate that TRIM24 bromodomain is an acetyllysine reader. Peptide pulldown assays and NMR titration measurements suggest that TRIM24 bromodomain interacts with $\mathrm{H} 3$ peptides with K23 or K27 acetylation and several acetylated H4 peptides (Supplementary Fig. 5a, b). ITC studies establish that TRIM24 PHD-Bromo specifically binds to the H3(13-32)K23ac peptide with a value of $K_{\mathrm{D}}(8.8 \mu \mathrm{M}$; Supplementary Table 2$)$ comparable to tetra-acetylated $\mathrm{H} 4$ peptide and double bromodomain modules of TAF1 or BDF1.

We solved the $1.9 \AA$ crystal structure of the complex of TRIM24 PHD-Bromo and H3(13-32)K23ac peptide (Supplementary Table 1 and Supplementary Fig. 6a). Residues 23-27 of the bound H3(1332)K23ac peptide exhibit sequence-specific interactions with TRIM24 bromodomain (Fig. $2 \mathrm{c}$ and Supplementary Fig. 6b). The acetyllysine side chain forms a direct hydrogen bond with the side chain of conserved N980. Acetyllysine recognition constitutes the binding determinant, as double mutant F979A/N980A loses most of the binding affinity for the H3(13-32)K23ac peptide (Supplementary Table 2).

ITC studies establish that $\mathrm{H} 3(1-20) \mathrm{K} 9 \mathrm{ac}, \mathrm{H} 3(1-19) \mathrm{K} 14 \mathrm{ac}$ and H3(13-32)K27ac bind non-specifically to the TRIM24 bromodomain $\left(K_{\mathrm{D}} \approx 200 \mu \mathrm{M}\right.$; Supplementary Table 2$)$. The crystal structure of the complex of TRIM24 PHD-Bromo with H3(23-31)K27ac peptide (Supplementary Table 1) revealed a single intermolecular hydrogen 

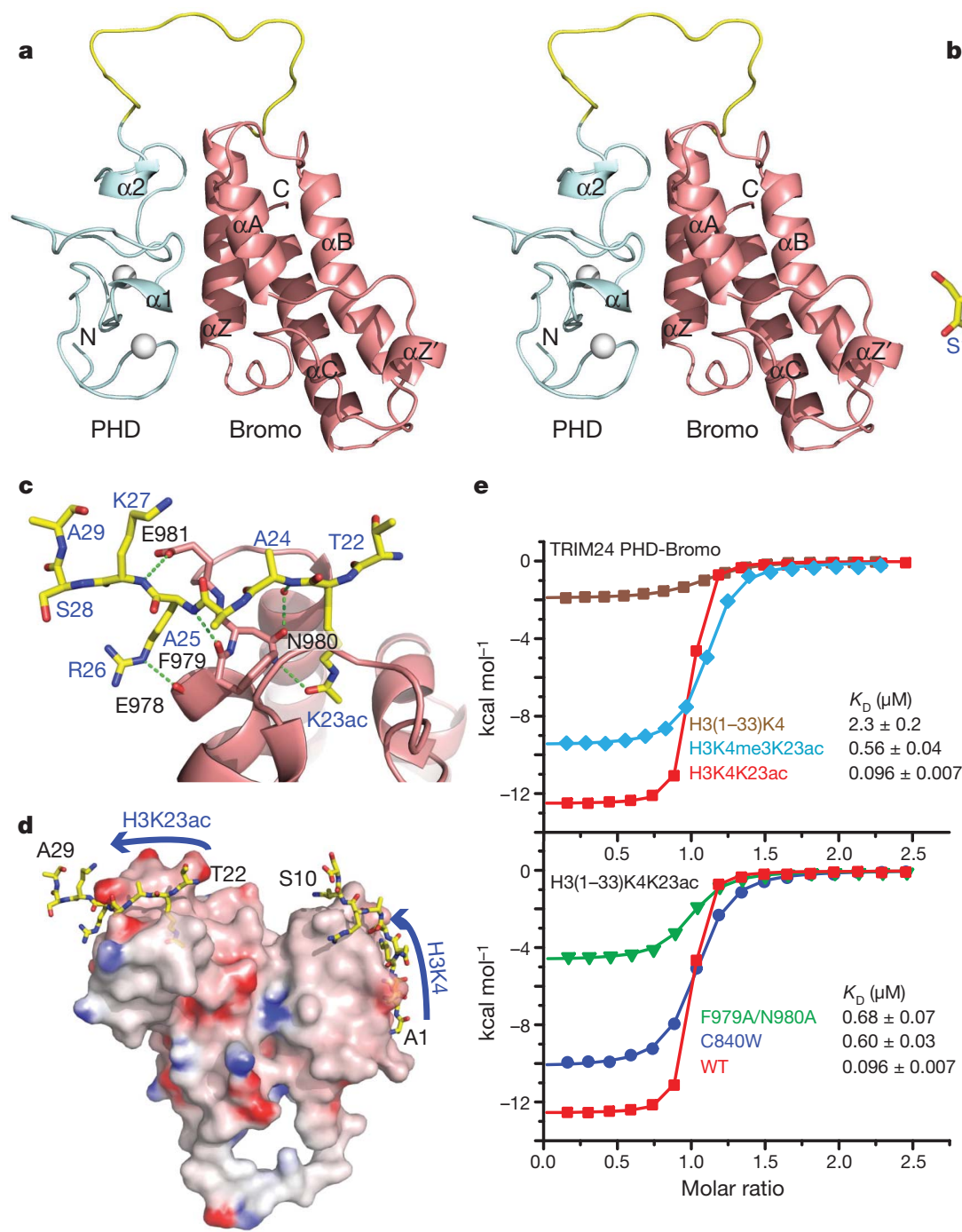

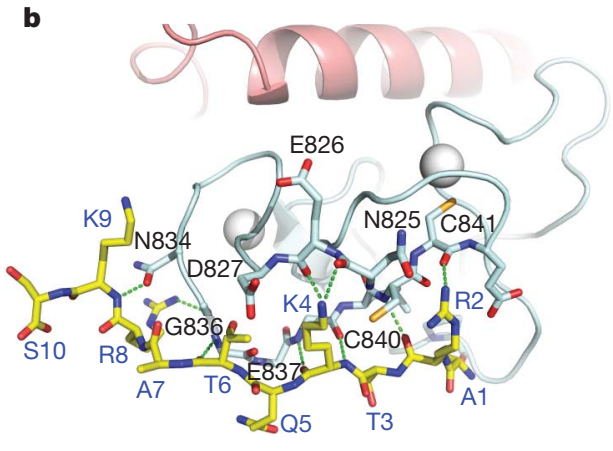

f

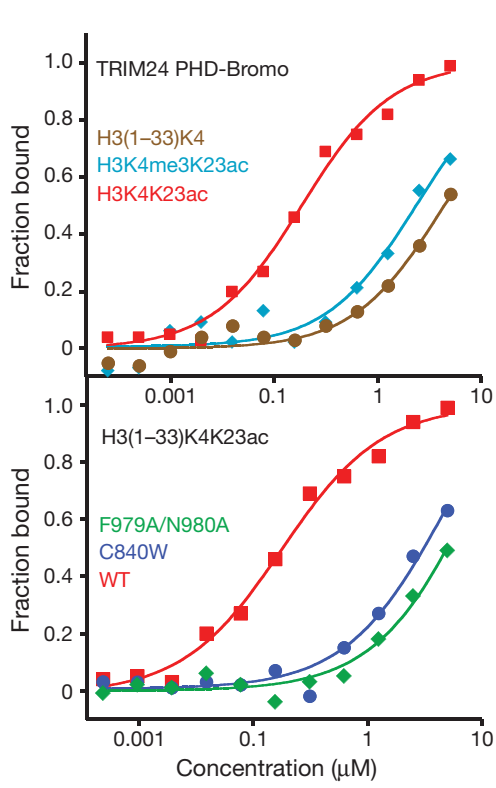

Figure 2 TRIM24 PHD-Bromo simultaneously binds H3K4me0 and acetylated histone lysines. a, Stereo view of the crystal structure of TRIM24 PHD-Bromo in the free state. b, Detailed interactions between PHD of TRIM24 PHD-Bromo and H3(1-10)K4 peptide. c, Detailed interactions between bromodomain of TRIM24 PHD-Bromo and H3(22-29)K23ac peptide. d, Positioning of H3(1-10)K4 and H3(13-32)K23ac peptides on the surface of

bond between the side chains of K27ac and N980, while other histone residues did not show any direct intermolecular contacts with the bromodomain (Supplementary Fig. 7), consistent with weak binding affinity of H3(13-32)K27ac peptide. The structure of TRIM24 PHDBromo bound to H4(14-19)K16ac peptide containing the conserved interaction between K16ac and N980 side chains is shown in Supplementary Fig. 8.

The structures of TRIM24 PHD-Bromo complexes with acetyllysinecontaining histone peptides show that acetyllysine invariantly inserts into a pre-formed acetyllysine-binding pocket of the bromodomain. With the acetyllysine as an anchor, flanking residues determine sequence specificity of acetyllysine peptides for the TRIM24 bromodomain. The H3(13-32)K23ac peptide both fits better within the cleft between ZA and BC loops, and shows sequence-specific interactions with TRIM24 bromodomain spanning K23ac to K27, creating much higher affinity for the TRIM24 bromodomain than is shown by other acetyllysine-containing peptides.

\section{Combinatorial readout by TRIM24 PHD-Bromo}

Superimposition of the above structures of complexes revealed that $\mathrm{H} 3 \mathrm{~K} 4$ and $\mathrm{H} 3 \mathrm{~K} 23 \mathrm{ac}$ peptides are aligned in the same direction on the
TRIM24 PHD-Bromo based on structural information. e, f, ITC (e) or fluorescence polarization (FP) (f) based binding curves of wild-type (WT) or mutant forms of TRIM24 PHD-Bromo with H3(1-33) peptides bearing different combination of modifications. Insets, $K_{\mathrm{D}}$ values derived from ITC experiments.

surface of the TRIM24 PHD-Bromo (Fig. 2d). The distance between the $\mathrm{C} \alpha$ of $\mathrm{H} 3 \mathrm{~K} 9$ and the $\mathrm{C} \alpha$ of $\mathrm{H} 3 \mathrm{~K} 23 \mathrm{ac}$ is $25.5 \AA$, which allows one $\mathrm{H} 3$ peptide containing both unmodified $\mathrm{H} 3 \mathrm{~K} 4$ and $\mathrm{H} 3 \mathrm{~K} 23 \mathrm{ac}$ to simultaneously target the PHD and bromodomain binding sites on TRIM24 PHD-Bromo.

By contrast, $\mathrm{H} 3 \mathrm{~K} 4$ and $\mathrm{H} 3 \mathrm{~K} 27 \mathrm{ac}$ (or $\mathrm{H} 4 \mathrm{~K} 16 \mathrm{ac}$ ) peptides are aligned in opposite directions on the surface of TRIM24 PHDBromo (Supplementary Fig. 9), which indicates that the TRIM24 PHD-Bromo requires two histone tails, either within a single nucleosome or from an adjacent pair of nucleosomes, to simultaneously bind $\mathrm{H} 3 \mathrm{~K} 4$ and H3K27ac (or H4K16ac).

To test the effect of combinatorial readout of TRIM24 PHD-Bromo on histone $\mathrm{H} 3$ bearing unmodified $\mathrm{K} 4$ and acetylated K23 dual marks, we synthesized longer $\mathrm{H} 3(1-33)$ peptides bearing both unmodified K4 and acetylated K23 marks. For controls, we used H3(1-33)K4me3K23ac, as well as $\mathrm{H} 3(1-33) \mathrm{K} 4$ peptides that have only one effective histone mark for specific TRIM24 PHD-Bromo recognition. On the basis of ITC binding assays, TRIM24 PHD-Bromo showed an approximately 90 -fold higher binding affinity for $\mathrm{H} 3(1-33) \mathrm{K} 4 \mathrm{~K} 23 \mathrm{ac}$ peptide (Fig. 2e, $\left.K_{\mathrm{D}}=0.096 \mu \mathrm{M}\right)$ compared to the shorter $\mathrm{H} 3(1-15) \mathrm{K} 4$ peptide bearing only unmodified $\mathrm{K} 4\left(K_{\mathrm{D}}=8.6 \mu \mathrm{M}\right)$ or for the $\mathrm{H} 3(13-32) \mathrm{K} 23 \mathrm{ac}$ peptide 
bearing only acetylated $\mathrm{K} 23$ marks $\left(K_{\mathrm{D}}=8.8 \mu \mathrm{M}\right)$. Without acetylation on $\mathrm{K} 23$, the binding for $\mathrm{H} 3(1-33) \mathrm{K} 4$ is 24-fold weaker (Fig. 2e; $\left.K_{\mathrm{D}}=2.3 \mu \mathrm{M}\right)$; when $\mathrm{K} 4$ is tri-methylated, the binding for $\mathrm{H} 3(1-$ 33)K4me3K23ac is sixfold weaker (Fig. 2e; $K_{\mathrm{D}}=0.56 \mu \mathrm{M}$ ). Similarly, mutants that disrupt either the PHD finger binding pocket (C840W) or the bromodomain binding pocket (F979A/N980A) also decreased binding for $\mathrm{H} 3(1-33) \mathrm{K} 4 \mathrm{~K} 23 \mathrm{ac}$ peptide by $6-7$ fold (Fig. 2e and Supplementary Table 2).

On the basis of fluorescence polarization (FP)-based measurement, wild-type TRIM24 PHD-Bromo also showed strong binding affinity for $\mathrm{H} 3(1-33) \mathrm{K} 4 \mathrm{~K} 23 \mathrm{ac}$ peptide $\left(K_{\mathrm{D}}=0.185 \mu \mathrm{M}\right)$; peptides trimethylated at K4 or without acetylation at K23 displayed 13-23 fold weaker interaction (Fig. 2f and Supplementary Table 3). Mutation on the PHD finger binding pocket $(\mathrm{C} 840 \mathrm{~W})$ or the bromodomain binding pocket (F979A/N980A) showed similar decrease in binding affinities (Fig. $2 \mathrm{f}$ and Supplementary Table 3 ). These binding data strongly support our structural results, which indicate that unmodified $\mathrm{H} 3 \mathrm{~K} 4$ and acetylated $\mathrm{H} 3 \mathrm{~K} 23$ are a pair of natural histone marks targeted by TRIM24 PHD-Bromo that can be read in a combinatorial manner on a single histone peptide. This combinatorial readout can greatly increase the recruitment of TRIM24 to nucleosomes bearing these two marks.

\section{TRIM24 and ER $\alpha$ recruitment to chromatin}

Combinatorial histone modifications of unmethylated $\mathrm{H} 3 \mathrm{~K} 4$ alongside acetylated lysines have no straightforward interpretation by the models of chromatin modification and regulated activation or repression of transcription. We considered a model where TRIM24 regulates gene expression by specific binding to chromatin with non-canonical combinations of PTMs, and focused on co-regulation of ER $\alpha$. This was because in vitro interactions between TRIM24 and nuclear receptors, including $\mathrm{ER} \alpha$, are ligand-dependent (Supplementary Fig. 10 and ref. 26), and because ligand-activated, ER-response elements (EREs) are notably independent of $\mathrm{H} 3 \mathrm{~K} 4 \mathrm{me} 2$ and $\mathrm{H} 3 \mathrm{~K} 4 \mathrm{me} 3$ modifications ${ }^{27,28}$. We used chromatin immunoprecipitation (ChIP) and sequential ChIP analyses of ER $\alpha$-positive, MCF7 breast cancer cells to assess whether TRIM24 is recruited with ER $\alpha$ to specific EREs of the GREB1, PR and pS2/TFF1 genes (Fig. 3a, b, and Supplementary Fig. 11). Oestrogenactivated recruitment occurs robustly within $15 \mathrm{~min}$, and by six hours yields a sevenfold increase of ER $\alpha$ binding and a sixfold increase of TRIM24 binding at the GREB1 distal ERE, $\sim 40$ kilobases (kb) upstream of the transcription start site (Fig. 3a). ChIP analysis of $\mathrm{H} 3 \mathrm{~K} 4 \mathrm{me} 2 / 3$ after oestrogen treatment indicates that quantified H3K4me2 and H3K4me3 levels decreased at distal ERE sites (Supplementary Fig. 12 and ref. 27) and, when normalized for nucleosomal occupancy, decreased or was unchanged at distal EREs (Fig. 3c and Supplementary Fig. 13). Importantly, TRIM24 is recruited in the absence of changes in $\mathrm{H} 3 \mathrm{~K} 4$ methylation. In contrast, $\mathrm{H} 3 \mathrm{~K} 23 \mathrm{ac}$, $\mathrm{H} 3 \mathrm{~K} 27 \mathrm{ac}$ and $\mathrm{H} 4 \mathrm{ac}$, which are targeted by the TRIM24 bromodomain, are enriched at both distal and proximal EREs after oestrogen-addition (Fig. 3d). These findings suggest that TRIM24 interacts with ER $\alpha$ and chromatin lacking H3K4 methylation but enriched in lysine acetylation, as suggested by our structural analyses, in response to oestrogen.

These findings stand in contrast to a model of chromatin accessibility at ER binding sites, facilitated by FOXA1 and H3K4me 2 enrichment in response to oestrogen treatment ${ }^{29}$, but are in agreement with findings that $\mathrm{H} 3 \mathrm{~K} 4 \mathrm{me} 3$ is not present at a majority of distal ERE regions ${ }^{28}$. We evaluated global chromatin-association of TRIM24, ER $\alpha$ and H3K4me2, by ChIP and deep sequencing of antibody-enriched DNA fragments (ChIP-seq). These analyses revealed binding of TRIM24 and ER at more than 10,000 sites genome-wide; half of which, in each case, are oestrogen-dependent (Fig. 3e and Supplementary Fig. 14a). Shared target sites of ER $\alpha$ and co-regulator TRIM24 increase dramatically (eightfold) in response to oestrogen (Supplementary Fig. 14b), are highly enriched $(P$ value $<0.001)$ at genes regulated by oestrogen ${ }^{30}$ (Supplementary Fig. 14c), and function in cell cycle, kinase activity
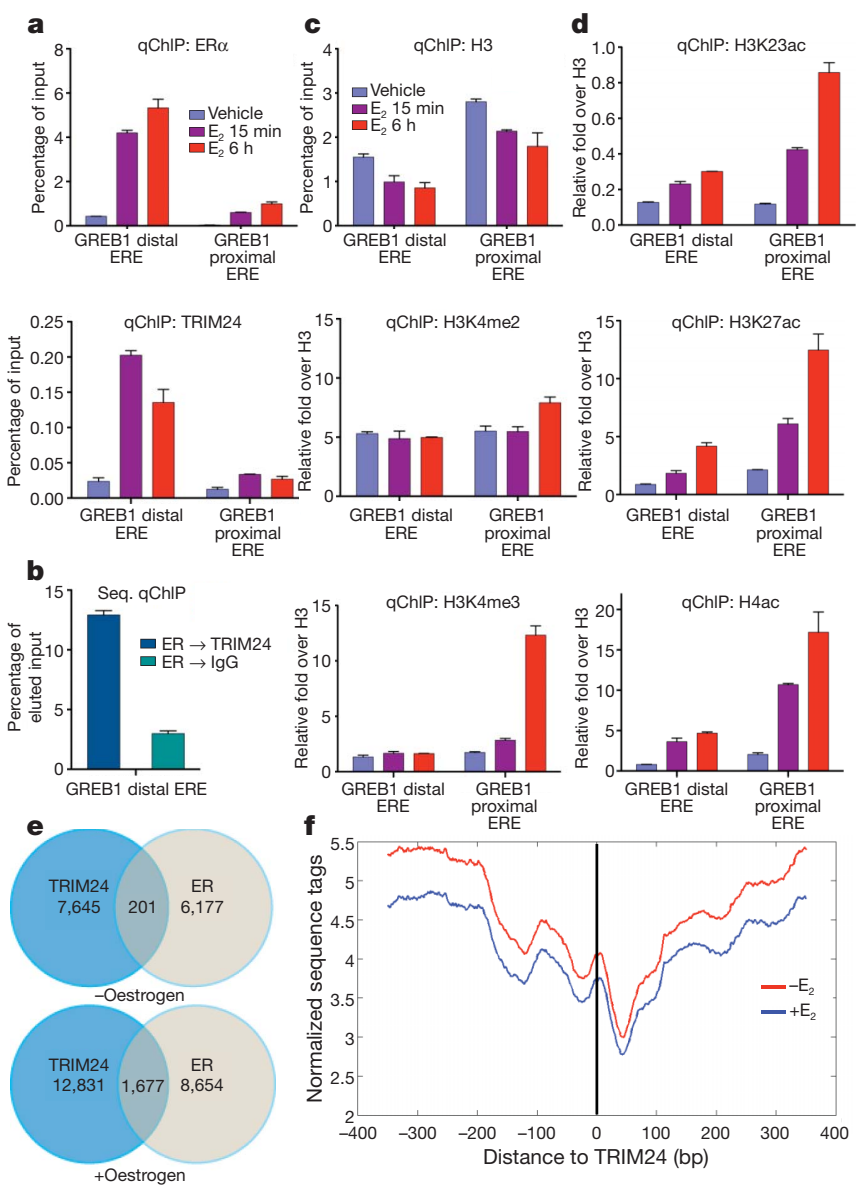

Figure 3 TRIM24 is recruited with ER $\alpha$ to ERE sites depleted of H3K4me2. a, ChIP of ER $\alpha$ and TRIM24 binding at EREs of GREB1, 15 min and $6 \mathrm{~h}$ after treatment with oestradiol $\left(\mathrm{E}_{2}\right)$. Vehicle, EtOH. b, Sequential-ChIP: ER $\alpha$ and TRIM24, $6 \mathrm{~h}$ after $\mathrm{E}_{2}$ addition. c, d, ChIP for $\mathrm{H} 3$ and histone modifications, $15 \mathrm{~min}$ and $6 \mathrm{~h}$ after $\mathrm{E}_{2}$, normalized for $\mathrm{H} 3$. Each bar represents averaged results, $n=3$ biological replicates, assayed 3 times each; error bars, s.d. e, Genome-wide TRIM24 and ER $\alpha$ binding sites in MCF7 cells, $-E_{2}$ or $+E_{2}$. Two independent experiments analysed. f, Normalized genome-wide $\mathrm{H} 3 \mathrm{~K} 4 \mathrm{me} 2$ within a window of $800 \mathrm{bp}$, centred at TRIM24 binding sites (designated as 0$),+\mathrm{E}_{2}$ (blue line) or $-\mathrm{E}_{2}$ (red line).

and signal transduction (DAVID analyses ${ }^{31}$, Supplementary Table 4). Biological pathway analysis (Ingenuity Systems, www.ingenuity.com) revealed that multiple gene targets of TRIM24 are associated with breast cancer (Supplementary Tables 5 and 6). The number of target sites shared by TRIM24 and ER $\alpha$ (1,677 sites) is similar to ER $\alpha$ and FOXA1 (ref. 29), with little overlap among all three (263 sites) (Supplementary Fig. 14b). Consistent with our structural analyses, TRIM24 binding occurs globally at sites depleted of H3K4me2 (Fig. 3f and Supplementary Figs 14d and 15). Thus, ER $\alpha$-regulated genes may be divided into multiple classes, defined by specific coregulators and their dependence on $\mathrm{H} 3 \mathrm{~K} 4$ methylation.

\section{TRIM24 is overexpressed in breast cancer}

Depletion of TRIM24 caused a significant decrease in ER $\alpha$-mediated activation of GREB1, PR and $p S 2$ gene expression (Fig. 4a and Supplementary Fig. 16a). Importantly, re-introduction of wild type (WT), but not PHD finger mutant (C840W), TRIM24 fully restored ER $\alpha$ mediated transcription activation (Fig. 4b), and enabled ER $\alpha$-response at lower levels of hormone (Fig. 4c). Decreased ER $\alpha$-mediated activation is due to loss of TRIM24-dependent ER $\alpha$-interactions with chromatin (Fig. 4d and Supplementary Fig. 17), without alternation of ER $\alpha$ expression (Supplementary Fig. 16b). H3K4me2/3 levels at the distal ERE of GREB1 lack hormone responsiveness and are TRIM24-independent 

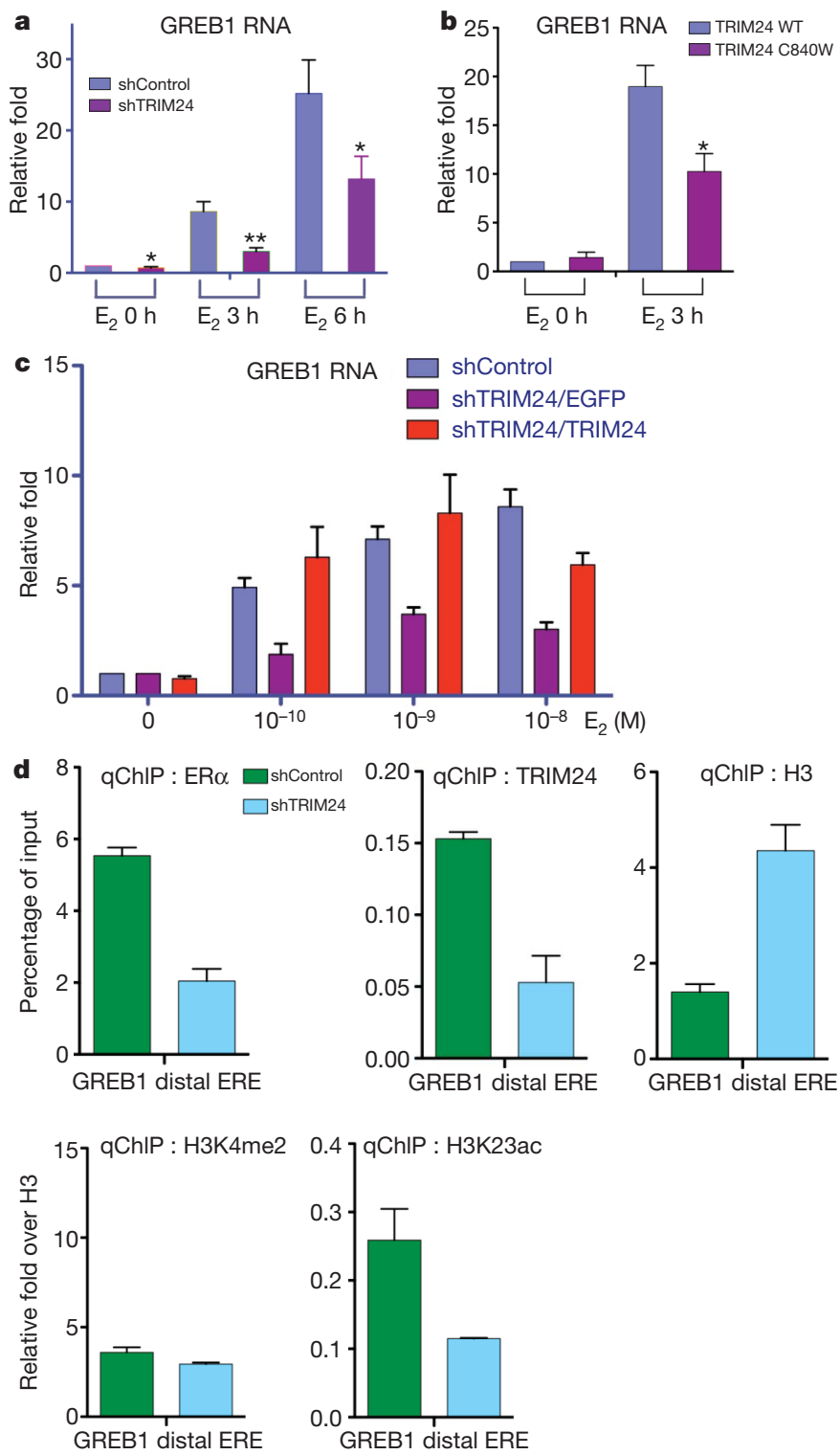

Figure 4 TRIM24 functions as a co-activator and stabilizes ER $\alpha-$ chromatin interactions. a, Stable shControl and shTRIM24 MCF7 cells $\pm E_{2}$. $* P<0.05$; ${ }^{*} P<0.01$. b, TRIM24 WT and TRIM24 C840W expressed in stable shTRIM24 MCF7 cells $\pm \mathrm{E}_{2}$. c, shControl and shTRIM24 MCF7 cells, $\mathrm{E}_{2}$ range. TRIM24 WT or EGFP control expressed in shTRIM24 MCF7 cells. In a, $\mathbf{b}$ and $\mathbf{c}, G R E B 1$ RNA levels are normalized to GAPDH; untreated shControl MCF7 is set as one. Each bar is an average of 3 biological replicates, 3 independent RT-PCR assays of each; error bars, s.d. d, ChIP of ER $\alpha$ and TRIM24 binding, histone $\mathrm{H} 3$ and histone modifications, $6 \mathrm{~h}$ after $\mathrm{E}_{2}$ addition, shControl and shTRIM24 MCF7 cells. Histone modifications normalized for $\mathrm{H} 3$ recovery. Each bar represents averaged results, $n=3$ and 3 assays of each; error bars, s.d.

(Fig. 4d and Supplementary Fig. 16c). In contrast, nucleosomal occupancy at EREs is increased alongside decreased acetylation of H4, $\mathrm{H} 3 \mathrm{~K} 23$ and $\mathrm{H} 3 \mathrm{~K} 27$, reflecting loss of $\mathrm{ER} \alpha$-activated chromatin structure (Fig. 4d and Supplementary Fig. 16c).

Depletion of TRIM24 led to reduced survival and proliferation of tumour-derived breast cancer cells, and is highly additive with 4-OHtamoxifen, an inhibitor of $\mathrm{ER} \alpha^{32}$ (Fig. 5a). We immunostained tissue samples from a breast cancer patient cohort to assess the impact of TRIM24 expression on breast cancer survival (Fig. 5b). In 128 cases of non-metastatic breast cancer, expression of TRIM24 fell into four classes: $\mathrm{N}-$ and $\mathrm{N}+$, undetectable to low level in few foci $(29 \%)$; $\mathrm{N}++$, abundant foci with expression in nuclear and cytoplasmic
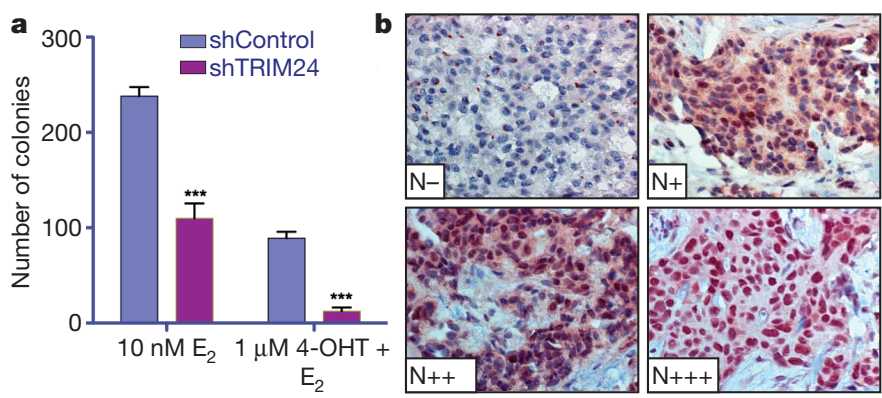

c

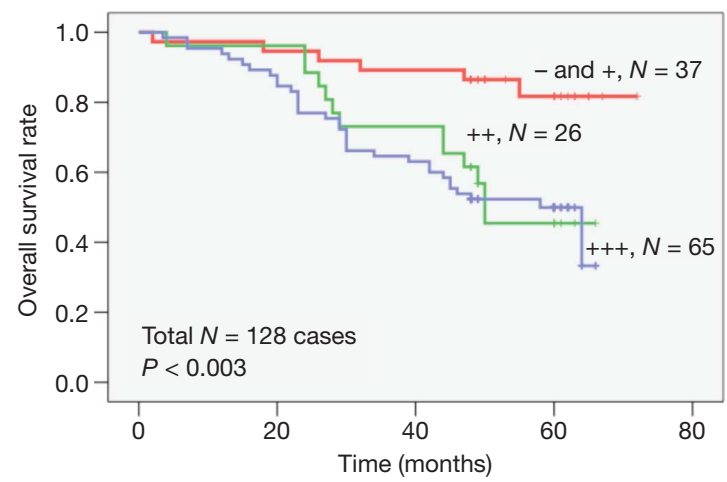

Figure 5 | Aberrant expression of TRIM24 correlates with poor survival of breast cancer patients. a, shControl and shTRIM24 MCF7 cells with $\mathrm{E}_{2}$ or $\mathrm{E}_{2}$ $+4-\mathrm{OH}$-tamoxifen (4-OHT), as indicated. Each bar represents the averaged results for three independent colony formation assays in triplicate plates; error bars, s.d. ${ }^{*} P<0.0001$. b, Immunohistochemistry: 128 surgical specimens of breast cancer immunostained for TRIM24: subcellular localization $(\mathrm{N})$ and staining intensity (strong, +++ ; moderate, ++ ; weak or slightly above background, +; none, - ). c, The overall survival rate of 128 patients with nonmetastatic disease, classified by TRIM24 expression (as in b), plotted by the Kaplan-Meier method.

compartments (20\%); and $\mathrm{N}+++$, abundant foci with high expression in nuclei $(51 \%)$. Overexpression of TRIM24 $(+++,++)$ is clearly correlated with poor patient survival, independent of ER status (Fig. 5c and Supplementary Table 7).

\section{Discussion}

Our identification of the PHD-Bromo as a reader of H3K4me0 and $\mathrm{H} 3 \mathrm{~K} 23 \mathrm{ac}$ within a single histone tail, or of $\mathrm{H} 3 \mathrm{~K} 4 \mathrm{me} 0$ and noncontiguous acetylated lysines, suggests that TRIM24 may have multiple roles in chromatin regulation ${ }^{20}$. TRIM24 is a co-activator of ER $\alpha$ at distal EREs, a platform well suited for stable interactions with TRIM24 PHD-Bromo. ER $\alpha$ recruits histone acetyltransferases-for example, CBP/p300, GCN5 and P/CAF (ref. 33) - to acetylate histones. LSD1 (KDM1), a biochemically and structurally characterized demethylase for H3K4me2/1 (refs 34, 35) and an androgen-regulated demethylase of $\mathrm{H} 3 \mathrm{~K} 9 \mathrm{me}$ (ref. 36 ), is resident ${ }^{37}$ or rapidly recruited ${ }^{27}$ to EREs where $\mathrm{H} 3 \mathrm{~K} 4$ remains depleted of methylation even with oestrogen activation (Fig. 3c, Supplementary Fig. 18 and ref. 28). These parallel processes establish a combinatorial histone signature with high affinity for TRIM24 binding to chromatin.

Aberrant expression of TRIM24 may promote tumour development and progression by multiple mechanisms of dysfunction. TRIM24 is a potent co-activator of $\mathrm{ER} \alpha$, which is associated with cellular proliferation and neoplasia in breast cells $\mathrm{s}^{38,39}$, and a negative regulator of p53 stability ${ }^{19}$. TRIM24 is a target of chromosomal translocations to form oncogenic fusion proteins in acute promyelocytic leukaemia ${ }^{40}$, papillary thyroid carcinoma ${ }^{41}$ and myeloproliferative syndrome $^{42}$. Here we have shown that TRIM24 expression is directly correlated with poor patient survival in both ER-positive and ERnegative breast cancer. These results suggest that TRIM24 is a dual 
domain histone reader with considerable potential as a therapeutic target in multiple cancers.

\section{METHODS SUMMARY}

Wild-type and mutant forms of TRIM24 PHD-Bromo were expressed in Escherichia coli and purified to homogeneity. Histone biotinylated peptides or purified histone proteins were incubated with GST-proteins, and bound proteins detected by immunoblotting. All crystals were obtained by the hanging-drop method at $20^{\circ} \mathrm{C}$; structures were solved by the molecular replacement method, and refined with cycled model building and refinement procedures. Histone peptides with or without biotin labelling were used for ITC binding. Fluorescein-labelled peptides were used for fluorescence polarization analysis. Stable short hairpin (sh)Control and shTRIM24 MCF7 cells were maintained with $2.5 \mu \mathrm{g} \mathrm{ml}^{-1}$ puromycin and, for hormone treatment, were grown in hormone-free media for $96 \mathrm{~h}$ before addition of ethanol or $10 \mathrm{nM}$ oestradiol (Sigma) for indicated times. Global expression analyses and calculation of enrichment of shared TRIM24 and ER $\alpha$ binding at oestrogen-regulated genes ${ }^{30}$ were determined, and validated by real-time RT-PCR. Surgical specimens of breast cancer from 128 non-metastatic patients were immunostained for TRIM 24 (TRIM24 antibody, Proteintech Group), and scored by subcellular localization, staining intensity, and fraction of positive staining. The overall survival after surgery was plotted by the Kaplan-Meier method ${ }^{43}$.

Full Methods and any associated references are available in the online version of the paper at www.nature.com/nature.

\section{Received 29 January; accepted 30 September 2010.}

1. Taverna, S. D. et al. How chromatin-binding modules interpret histone modifications: lessons from professional pocket pickers. Nature Struct. Mol. Biol. 14, 1025-1040 (2007)

2. Jenuwein, T. \& Allis, C. D. Translating the histone code. Science $\mathbf{2 9 3 , 1 0 7 4 - 1 0 8 0}$ (2001).

3. Ruthenburg, A. J., Li, H., Patel, D. J. \& Allis, C. D. Multivalent engagement of chromatin modifications by linked binding modules. Nature Rev. Mol. Cell Biol. 8 , 983-994 (2007).

4. Berger, S. L. The complex language of chromatin regulation during transcription. Nature 447, 407-412 (2007)

5. Baker, L. A., Allis, C. D. \& Wang, G. G. PHD fingers in human diseases: disorders arising from misinterpreting epigenetic marks. Mutat. Res. 647, 3-12 (2008).

6. Jacobson, R. H., Ladurner, A. G. King. D. S. \& Tijian, R. Structure and function of a human TAFII250 double bromodomain module. Science 288, 1422-1425 (2000).

7. Morinière, J. etal. Cooperative binding of two acetylation marks on a histone tail by a single bromodomain. Nature 461, 664-668 (2009)

8. Lan, F. et al. Recognition of unmethylated histone H3 lysine 4 links BHC80 to LSD1-mediated gene repression. Nature 448, 718-722 (2007).

9. Org, T. et al. The autoimmune regulator PHD finger binds to non-methylated histone H3K4 to activate gene expression. EMBO Rep. 9, 370-376 (2008).

10. Ragvin, A. et al. Nucleosome binding by the bromodomain and PHD finger of the transcriptional cofactor p300. J. Mol. Biol. 337, 773-788 (2004).

11. Zhou, Y. \& Grummt, I. The PHD finger/bromodomain of NoRC interacts with acetylated histone H4K16 and is sufficient for rDNA silencing. Curr. Biol. 15, 1434-1438 (2005)

12. Peña, P. V. et al. Molecular mechanism of histone H3K4me3 recognition by plant homeodomain of ING2. Nature 442, 100-103 (2006).

13. Li, H. et al. Molecular basis for site-specific read-out of histone H3K4me3 by the BPTF PHD finger of NURF. Nature 442, 91-95 (2006).

14. Lange, M. et al. Regulation of muscle development by DPF3, a novel histone acetylation and methylation reader of the BAF chromatin remodeling complex. Genes Dev. 22, 2370-2384 (2008).

15. Koh, A. S. etal. Aire employs a histone-binding module to mediate immunologica tolerance, linking chromatin regulation with organ-specific autoimmunity. Proc. Natl Acad. Sci. USA 105, 15878-15883 (2008).

16. Hung, T. et al. ING4 mediates crosstalk between histone $\mathrm{H} 3 \mathrm{~K} 4$ trimethylation and H3 acetylation to attenuate cellular transformation. Mol. Cell 33, 248-256 (2009).

17. Wang, Z. etal. Pro isomerization in MLL1 PHD3-bromo cassette connects H3K4me readout to CyP33 and HDAC-mediated repression. Cell 141, 1183-1194 (2010).

18. Wang, G. G. et al. Haematopoietic malignancies caused by dysregulation of a chromatin-binding PHD finger. Nature 459, 847-851 (2009).

19. Allton, K. et al. Trim 24 targets endogenous $p 53$ for degradation. Proc. Natl Acad. Sci. USA 106, 11612-11616 (2009).

20. Poleshko, A. et al. Identification of a functional network of human epigenetic silencing factors. J. Biol. Chem. 285, 422-433 (2009).

21. Meroni, G. \& Diez-Roux, G. TRIM/RBCC, a novel class of 'single protein RING finger' E3 ubiquitin ligases. Bioessays 27, 1147-1157 (2005).

22. Reymond, A. et al. The tripartite motif family identifies cell compartments. EMBO J. 20, 2140-2151 (2001)

23. Le Douarin, B.etal.Apossible involvementofTIF1 alphaand TIF1 betain theepigenetic control of transcription by nuclear receptors. EMBO J. 15, 6701-6715 (1996).
24. Soliman, M. A. \& Riabowol, K. After a decade of study-ING, a PHD for a versatile family of proteins. Trends Biochem. Sci. 32, 509-519 (2007).

25. Peña, P. V. et al. Histone H3K4me3 binding is required for the DNA repair and apoptotic activities of ING1 tumor suppressor. J. Mol. Biol. 380, 303-312 (2008).

26. Thénot, S., Henriquet, C., Rochefort, H. \& Cavailles, V. Differential interaction of nuclear receptors with the putative human transcriptional coactivator hTIF1.J. Biol. Chem. 272, 12062-12068 (1997).

27. Garcia-Bassets, l. etal. Histone methylation-dependent mechanisms impose ligand dependency for gene activation by nuclear receptors. Cell 128, 505-518 (2007).

28. Fullwood, M. J. et al. An oestrogen-receptor- $\alpha$-bound human chromatin interactome. Nature 462, 58-64 (2009).

29. Lupien, M. et al. FoxA1 translates epigenetic signatures into enhancer-driven lineage-specific transcription. Cell 132, 958-970 (2008)

30. Lin, C. Y. et al. Whole-genome cartography of estrogen receptor $\alpha$ binding sites. PLoS Genet. 3, e87 (2007).

31. Huang, W, Sherman, B. T. \& Lempicki, R. A. Systematic and integrative analysis of large gene lists using DAVID bioinformatics resources. Nature Protocols 4, 44-57 (2009).

32. Eckert, R. L. \& Katzenellenbogen, B. S. Physical properties of estrogen receptor complexes in MCF-7 human breast cancer cells. Differences with anti-estrogen and estrogen. J. Biol. Chem. 257, 8840-8846 (1982).

33. Yanagisawa, J. et al. Nuclear receptor function requires a TFTC-type histone acetyl transferase complex. Mol. Cell 9, 553-562 (2002)

34. Stavropoulos, P., Blobel, G. \& Hoelz, A. Crystal structure and mechanism of human Iysine-specific demethylase-1. Nature Struct. Mol. Biol. 13, 626-632 (2006).

35. Shi, Y. et al. Histone demethylation mediated by the nuclear amine oxidase homolog LSD1. Cell 119, 941-953 (2004).

36. Wissmann, M. et al. Cooperative demethylation by JMJD2C and LSD1 promotes androgen receptor-dependent gene expression. Nature Cell Biol. 9,347-353 (2007).

37. Perillo, B. et al. DNA oxidation as triggered by $\mathrm{H} 3 \mathrm{~K} 9 \mathrm{me} 2$ demethylation drives estrogen-induced gene expression. Science 319, 202-206 (2008).

38. Katzenellenbogen, B. S. Estrogen receptors: bioactivities and interactions with cell signaling pathways. Biol. Reprod. 54, 287-293 (1996).

39. Cheskis, B. J., Greger, J. G., Nagpal, S. \& Freedman, L. P. Signaling by estrogens. J. Cell. Physiol. 213, 610-617 (2007).

40. Zhong, S. et al. A RA-dependent, tumour-growth suppressive transcription complex is the target of the PML-RAR $\alpha$ and T18 oncoproteins. Nature Genet. 23, 287-295 (1999).

41. Klugbauer, S. \& Rabes, H. M. The transcription coactivator HTIF1 and a related protein are fused to the RET receptor tyrosine kinase in childhood papillary thyroid carcinomas. Oncogene 18, 4388-4393 (1999).

42. Belloni, E. etal. $8 \mathrm{p} 11$ myeloproliferative syndrome with a novel $\mathrm{t}(7 ; 8)$ translocation leading to fusion of the FGFR1 and TIF1 genes. Genes Chromosom. Cancer 42, 320-325 (2005).

43. Xia,W.etal.Phosphorylation/cytoplasmic localization of 21 Cip1/WAF1 is associated with HER2/neu overexpression and provides a novel combination predictor for poor prognosis in breast cancer patients. Clin. Cancer Res. 10, 3815-3824 (2004).

Supplementary Information is linked to the online version of the paper at www.nature.com/nature.

Acknowledgements This work was supported by funds from the National Institutes of Health (NIH GM081627) and the George and Cynthia Mitchell Foundation (to M.C.B.), from NIH (U54 RRO25216 and P30DK078392-01) to B.A., from NIH (GM079641) to $0 . G$., from the Sister Institution Fund of China Medical University and Hospital and MDACC to M.-C.H., from the Starr Foundation and the Leukemia and Lymphoma Society to D.J.P., from the Max Planck Society to W.F., and from the NCl Cancer Center (Support Grant) to the UT MD Anderson Cancer Center. W.-W.T. was supported in part by the Sowell-Huggins Foundation; S.W. by a long-term EMBO fellowship; T.T.Y. by T32 HD07325; and K.C.A. by the Center for Cancer Epigenetics. We thank J. Song, D.C. Jamison, A. Dose, Z. Coban and Y. Wei for technical support and assistance. We are grateful to S. Stratton, M. Lee, M. Bedford, G. Lozano, S. Dent, A. Nardulli and members of our laboratories for advice, reagents and discussions.

Author Contributions W.-W.T. identified ER interactions, and performed molecular biology and IHC studies: Z.W. solved the molecular structures of TRIM24 PHD-Bromo in the free and bound states, and performed ITC binding affinity studies; T.T.Y. performed mutagenesis, ChIP and clonogenic analyses; C.-Y.T. performed clonogenic assays; K.C.A. performed bioinformatic analyses; W.X. analysed patient samples; X.S. performed peptide array analyses; S.W., D.S. and W.F. performed and analysed FP experiments; O.G., B.A., W.P., W.F., M.-C. H., D.J.P. and M.C.B. discussed studies; and D.J.P. and M.C.B. designed structural and functional studies, analysed data and wrote the paper. W.-W.T. and Z.W. contributed equally to this work. All authors discussed and commented on the manuscript.

Author Information The X-ray coordinates of TRIM24 PHD-Bromo in the free state and when bound to H3(1-10)K4, H3(13-32)K23ac, H3(23-31)K27ac and H4(1419)K16ac peptides have been deposited in the Protein Data Bank (PDB) under accession numbers $3033,3037,3034,3035$ and 3036 , respectively.

ChIP-sequencing files and data are deposited at the NCBI Gene Expression Omnibus (GEO) site as accession number GSE24166. Reprints and permissions information is available at www.nature.com/reprints. The authors declare no competing financial interests. Readers are welcome to comment on the online version of this article at www.nature.com/nature. Correspondence and requests for materials should be addressed to D.J.P. (pateld@mskcc.org) or M.C.B. (mbarton@mdanderson.org). 


\section{METHODS}

Cell culture. MCF7 and HEK293T cells were obtained from ATCC. Stable shControl and shTRIM24 MCF7 cells were maintained with $2.5 \mu \mathrm{g} \mathrm{ml}^{-1}$ puromycin. For hormone treatment, MCF7 cells were plated under normal growth condition (DMEM with $10 \%$ FBS and $1 \times$ penicillin/streptomycin). When cell density reached $25 \%$ confluence, the media were changed to hormone-free media (phenol red-free DMEM, 10\% charcoal dextran-treated FBS) for $96 \mathrm{~h}$. The hormone-deprived MCF7 cells were treated with ethanol or $10 \mathrm{nM}$ oestradiol (Sigma) for indicated times. The TRIM24 shRNA for stable cell line creation was AAGCA GGTGG AACAG GATAT TAAAG TTGC.

Biotinylated peptide pulldown assay. Histone biotinylated peptides $(1 \mu \mathrm{g}$, Millipore) were incubated with GST-proteins $(2 \mu \mathrm{g})$, in $500 \mu \mathrm{l} \mathrm{NTP} \mathrm{buffer}$ $(300 \mathrm{mM} \mathrm{NaCl}, 50 \mathrm{mM}$ Tris- $\mathrm{HCl}, 0.1 \% \mathrm{NP}-40, \mathrm{pH} 7.4)$ overnight at $4{ }^{\circ} \mathrm{C} .20 \mu \mathrm{l}$ of a $50 \%$ slurry of Streptavidin-coated beads (Thermo) were added and incubated for $1 \mathrm{~h}$ at $4{ }^{\circ} \mathrm{C}$. The beads were recovered by centrifugation and washed 6 times $\left(10 \mathrm{~min}\right.$ at $4{ }^{\circ} \mathrm{C}$ ) with NTP. The peptide/protein bound beads were resuspended in $3 \times$ SDS-PAGE loading buffer, heated for $5 \mathrm{~min}$ at $95^{\circ} \mathrm{C}$ and separated on $4-12 \%$ gradient polyacrylamide gels (Invitrogen). GST-proteins were detected by western blotting analysis with anti-GST antibody (Cell Signaling, dilution 1:1,000).

GST protein pull-down assay, co-IP and transient transfection. GST-proteins $(2 \mu \mathrm{g})$ were incubated with purified histone proteins $(10 \mu \mathrm{g})$ in $500 \mu \mathrm{l} \mathrm{NTP}$ overnight at $4{ }^{\circ} \mathrm{C} .25 \mu \mathrm{l}$ of a $50 \%$ slurry of GST-beads were added and incubated for $2 \mathrm{~h}$ at $4{ }^{\circ} \mathrm{C}$, recovered by centrifugation and washed 4 times $\left(10 \mathrm{~min}\right.$ at $\left.4{ }^{\circ} \mathrm{C}\right)$ with NTP. The protein bound beads were separated by SDS-PAGE and subjected to western blotting analysis. Anti-GST (Cell Signaling), anti-TRIM24 (Novus Biological), anti-histone H3 (Abcam), anti-H3K9me2 (Active Motif) and antiH3K4me3 (Active Motif) antibodies were used in immunoblotting.

Ethidium bromide-treated and precleared cell lysate was used for IP with TRIM24 antibody ( $2.5 \mu \mathrm{l}$, Novus Biological) or control rabbit IgG antibody $\left(2.5 \mu \mathrm{l}\right.$, Upstate) as previously described ${ }^{44}$. Then anti-ER (F-10, Santa Cruz) and anti-TRIM24 were used in western blotting analysis. Transient transfections were performed as previously described ${ }^{45}$, but with Effectene (Qiagen) used according to manufacturer's directions.

Chromatin immunoprecipitation assay (ChIP). ChIP assays were performed as described ${ }^{45}$, with minimal modification. Briefly, treated MCF7 cells were crosslinked with $1 \%$ formaldehyde for $15 \mathrm{~min}$. The cross-linking reaction was stopped with $0.125 \mathrm{M}$ glycine. The cross-linked cells were washed with PBS three times and stored at $-80^{\circ} \mathrm{C}$ before use. The fragmented, precleared chromatin lysate was incubated overnight with specific antibodies: ER (F-10, Santa Cruz), TRIM24 (Novus Biological), histone H3 (Abcam), H3K4me2 (Active Motif), H3K4me3 (Active Motif), H3K23ac (Active Motif), H3K27ac (Active Motif), H4ac (Upstate/Millipore) or normal sheep IgG (Upstate/Millipore). To analyse specific, antibody- and protein-bound DNA, qPCR was conducted in a 7500 FAST $\mathrm{ABI}$ instrument. PCR primers were used as described ${ }^{27,46}$.

ChIP-sequencing analysis. Purified ChIP DNA was prepared for sequencing using the Illumina ChIP-Seq sample preparation kit (IP-102-1001, Illumina). Sequencing was performed on an Illumina Genome Analyser II using 36 cycles. Sequences were aligned to human genome release hg18 (ref. 47) using the ELAND software at the default 0 to 2 allowed mismatch setting ${ }^{48}$.

High-quality aligned read data was converted to the BED format and analysed for peaks using the MACS software programs ${ }^{49}$. Peaks showing altered pull-down patterns were mapped to the genome, and a list of candidate genes flanking each peak was generated. Foxal (ChIP-Chip) binding regions ${ }^{29}$ were obtained from http://research.dfci.harvard.edu/brownlab/datasets/. Overlapping regions were identified if any of two regions share more than $80 \%$ of their length with the other region. Venn diagrams were prepared on the basis of that assumption.

To identify possible target genes, a RefSeq data file was downloaded from UCSC genome browser (hg18). Genes within $10 \mathrm{~kb}$ of the binding regions were marked as target genes; DAVID ${ }^{31}$ was used to assess biological functions of targets, and Ingenuity Pathway Analysis (IPA) software was used to ascertain the pathways that are enriched by these target genes (Ingenuity Systems, www. ingenuity.com). For H3K4me2 signals around the Trim 24 binding regions, in the presence or absence of oestrogen, numbers of fragments that overlap each position were counted. The Input signal was calculated by the same procedure and overall H3K4me2 signal values were normalized by Input signal.

For intersection with global expression analyses and calculation of enrichment of shared Trim 24 and ER binding at oestrogen-regulated genes ${ }^{30}$, raw CEL files were downloaded from the GEO database. RMA normalization was used with default options (with background correction, quantile normalization, and log transformation) to normalize the intensity of each probeset. The SAM statistical method was used to select differentially expressed genes, based on a $q$-value of less than $2 \%$. After filtration, a total of 1,887 genes were selected as differentially expressed after oestrogen treatment. Venn diagrams were formed based on the proximity of binding regions to genes within $10 \mathrm{~kb}$ of the binding sites. $P$-values were calculated based on binomial tests.

Real time RT-PCR analysis. RNA was isolated at indicated time points using Trizol reagent (Invitrogen) following manufacturer's instructions. cDNA was synthesized using a RT-PCR kit (Invitrogen) according to manufacturer's instructions. Synthesized cDNA was treated with RNaseH before performing PCR reactions. Real time PCR reactions were performed in an Applied Biosystems 7500 Fast Real time PCR instrument. Generally, $10 \mu \mathrm{l}$ PCR reactions were set up in 96-well plates. The reaction mixtures contain $2 \mu \mathrm{l}$ diluted cDNA (1:10 dilution), $0.25 \mu \mathrm{l}$ each of forward and reverse primers $(20 \mu \mathrm{M}), 5 \mu \mathrm{l} 2 \times$ SYBR Green Mix and $2.5 \mu \mathrm{l}$ water. PCR primers were used as described ${ }^{46}$

GraphPad Prism5 software was used for analysis of $P$-values based on at least two independent experiments in three independent PCR reactions. The two-tailed paired $t$-test was used to compare the differences between two groups for relative fold. The two-tailed unpaired $t$-test was used to compare the differences between two groups for actual percentage. $P$-values $<0.05$ were considered statistically significant.

Colony formation assay. 500 stable control shRNA depleted or TRIM24 shRNA depleted MCF7 cells were distributed in DMEM with $10 \% \mathrm{FBS}$ and $1 \times \mathrm{P} / \mathrm{S}$ and then placed in $60 \mathrm{~mm}^{2}$ plates at $37^{\circ} \mathrm{C}$ for 14 days. Colonies were stained by crystal violet 14 days after seeding. Colonies of $>50$ cells were counted using a dissecting microscope. Each sample was performed in triplicate.

Clinical patient samples and immunohistochemistry. We obtained 134 archived blocks containing formalin-fixed, paraffin-embedded infiltrating breast carcinoma from the Department of Pathology, Shanghai East Breast Disease Hospital, China. All of the patients were women with non-metastatic disease who had undergone mastectomy and axillary lymph node dissection between 1988 and 1994. After surgical treatment, the patients were offered adjuvant chemotherapy and/or radiotherapy and hormone therapy, depending on the number of lymph node metastases, status of menopause, and oestrogen and/or progesterone receptor positivity. The clinicopathological characteristics of the study population, including age, tumour size, lymph node status, tumour grade, and oestrogen receptor/progesterone receptor positivity, were obtained from medical records. The stage was assessed by the TNM clinical staging system of the American Joint Committee on Cancer. Patients were followed for 4-72 months, with a median follow-up of 48 months. We have follow-up information from 128 patients. Most of the tissues in this cohort were described previously ${ }^{43}$, and used as approved by the Institutional Review Board.

128 surgical specimens of breast cancer were immunostained for TRIM 24 (TRIM24 antibody, Proteintech Group). Immunoreactivity of the TRIM24 antibody was scored according to the subcellular localization (nuclear and/or cytoplasmic), staining intensity (strong +++ , moderate ++ , weak + and faint or slightly above background), and fraction of positive staining. The mean fraction of positive tumour cells was determined in at least nine areas at $\times 100$ or $\times 200$ magnification. Immunoreactivity scores were as described previously. For example, 0 is no staining. + is weak staining; ++ is moderate staining; +++ is strong staining and the values for percentage of positive tumour cells: $0=0-0.9 \% ; 1=1-10 \% ; 2=11-50 \%$, $3=51-80 \% ; 4=81-100 \%$. Magnification was $\times 400$; at least 200 tumour cells were counted ${ }^{43}$. ER assessment was done in a similar manner.

Data on eligible patients were summarized by use of standard descriptive statistics and frequency tabulation. The overall survival after surgery was plotted by use of the Kaplan-Meier method. The log-rank test was used to analyse differences in survival time. All tests were two-sided and the level of significance was set at 0.05 .

Histone peptide microarray hybridization. Peptide microarray assays were performed as described ${ }^{50}$. Briefly, biotinylated histone peptides were printed in hexaplicates onto a streptavidin coated slide (ArrayIt) using a VersArray Compact Microarrayer (BioRad). After short blocking with biotin (Sigma), the slides were incubated with the GST-TRIM24 PHD-Bromo in binding buffer (300 mM NaCl, $50 \mathrm{mM}$ Tris- $\mathrm{HCl}$ 7.5, 0.1\% NP-40, 1 mM PMSF, 20\% fetal bovine serum) overnight at $4{ }^{\circ} \mathrm{C}$ with gentle agitation. After washing with the same buffer, slides were probed with anti-GST antibody and then fluorescein-conjugated secondary antibody and visualized with a GenePix 4000 scanner (Molecular Devices).

Protein preparation. As the long linker (30 residues) between TRIM24 PHD finger and bromodomain was predicted to be a loop, we first expressed PHD finger (824-886) and bromodomain (896-1016) separately, and then checked their interaction by the gel-filtration method. The mixture of PHD finger and bromodomain contained a higher molecular weight peak that corresponded to the complex fraction (Supplementary Fig. 16), which indicates that TRIM24 PHD-Bromo forms a structural unit with direct interactions between the domains. Next, we focused on the expression and crystallization of the TRIM24 PHD-Bromo dual domain. We cloned different lengths of TRIM24 
PHD-Bromo fragments into pRSFDuet-1 vector (modified pRSFDuet-1 vector with $6 \times$ His plus yeast sumo as fusion tag). Fragment 824-1011 was used for all the binding assays. Further, all the mutations were introduced into the same construct using a QuikChange Kit (Stratagene). Fragment 824-1006 was used for all crystallographic studies. Protein expression was carried out in BL21 (DE3) E. coli cells. Cells were grown until $A_{600}$ reached around 1.0, then the media were cooled and $0.2 \mathrm{mM}$ IPTG and $0.1 \mathrm{mM} \mathrm{ZnCl} 2$ were added to the culture to induce the protein expression at $25^{\circ} \mathrm{C}$ for $8 \mathrm{~h}$.

E. coli-expressed wild-type and mutant TRIM24 PHD-Bromo were affinity purified by nickel-charged HisTrap Chelating FF columns (GE Healthcare). E. coli cells were disrupted by sonication in loading buffer $(20 \mathrm{mM}$ Tris $(\mathrm{pH}$ 8.0), $500 \mathrm{mM} \mathrm{NaCl}, 20 \mathrm{mM}$ imidazole). After centrifugation, the supernatant was loaded onto a nickel column and was washed extensively with loading buffer. The target protein was eluted with a linear gradient of 50-500 mM imidazole. The target protein was collected and dialysed overnight in loading buffer, with Ulp1 protease added to cleave the $6 \times$ His-sumo tag. Digested protein was loaded onto a nickel-column again to remove the $6 \times$ His-sumo tag and the His-tagged protease. The flow through containing TRIM24 PHD-Bromo was collected and further purified by a Hiload $7526 / 60$ column in buffer containing $10 \mathrm{mM}$ Tris ( $\mathrm{pH} 8.0$ ), $100 \mathrm{mM} \mathrm{NaCl}$ and $5 \mathrm{mM}$ DTT. The major peak was pooled, concentrated to high concentration and stored in a $-80^{\circ} \mathrm{C}$ freezer.

Crystallization. Crystals of TRIM24 PHD-Bromo in the free form were grown by mixing equal volumes of $20 \mathrm{mg} \mathrm{ml}^{-1}$ protein and crystallization buffer $(100 \mathrm{mM}$ Hepes (pH 7.5), $2.0 \mathrm{M}$ ammonium sulphate and $2 \%$ polyethylene glycol 400 ) at $20{ }^{\circ} \mathrm{C}$. Crystals appeared after two days and reached their full lengths within a week.

Crystals of the complex of TRIM24 PHD-Bromo and unmodified H3(1-10)K4 peptide were grown by incubating $20 \mathrm{mg} \mathrm{ml}^{-1}$ protein with peptide at a $1: 1.5$ ratio, followed by mixing with an equal volume of crystallization buffer $(50 \mathrm{mM}$ Tris ( $\mathrm{pH} 7.5$ ), $30 \%$ polyethylene glycol monomethyl ether 5000 , and $100 \mathrm{mM}$ $\mathrm{NaCl}$ ) at $20^{\circ} \mathrm{C}$. Crystals appeared within a week and reached their full size in two weeks.

Crystals of the complex of TRIM24 PHD-Bromo and H3(13-32)K23ac peptide were grown by mixing $20 \mathrm{mg} \mathrm{ml}^{-1}$ protein with peptide at $1: 1.5$ ratio, followed by mixing with an equal volume of crystallization buffer $(100 \mathrm{mM}$ sodium citrate ( $\mathrm{pH}$ 5.6), $200 \mathrm{mM}$ potassium/sodium tartrate tetrahydrate and 1.6 $\mathrm{M}$ ammonium sulphate) at $20^{\circ} \mathrm{C}$.

Crystals of the complex of TRIM24 PHD-Bromo and H3(23-31)K27ac peptide were grown by mixing $20 \mathrm{mg} \mathrm{ml}^{-1}$ protein with peptide at a 1:3 ratio, followed by mixing with an equal volume of crystallization buffer ( $50 \mathrm{mM}$ Tris ( $\mathrm{pH} \mathrm{8.0)}$, $200 \mathrm{mM}$ sodium acetate, $30 \%$ polyethylene glycol 4000 ) at $20^{\circ} \mathrm{C}$. Initially, we could only get twinned crystals that could not be used for structure determination. Later, single crystals were obtained by including detergent $0.8 \mathrm{mM} \mathrm{CHAPSO}$ (Hampton Research) into the crystallization buffer.

Crystals of the complex of TRIM24 PHD-Bromo and H4(14-19)K16ac peptide were prepared by mixing $20 \mathrm{mg} \mathrm{ml}^{-1}$ protein with peptide at a 1:3 ratio, followed by mixing with an equal volume of crystallization buffer $(50 \mathrm{mM}$ Bis-Tris $(\mathrm{pH}$ 6.5), $30 \%$ polyethylene glycol $3350,100 \mathrm{mM}$ ammonium acetate) at $20^{\circ} \mathrm{C}$. Crystals appeared within a day and reached their full lengths in a week. Fresh crystals (grown within a week) were used for data collection, as crystals grown for longer than 3 weeks diffracted poorly. For all the crystals mentioned above, crystallization buffers with $20 \%$ glycerol were used as cryoprotectant.

Data collection and structure determination. Data sets for crystals of TRIM24 PHD-Bromo in the free state, as well as TRIM24 PHD-Bromo complexes with unmodified $\mathrm{H} 3(1-10) \mathrm{K} 4$, with $\mathrm{H} 3(13-32) \mathrm{K} 23 \mathrm{ac}$ and with $\mathrm{H} 4(14-19) \mathrm{K} 16 \mathrm{ac}$ peptides were collected at NE-CAT beamline 24ID-E, Advanced Photon Source, Chicago. Data sets for crystals of the complex of TRIM24 PHD-Bromo and H3(23-31)K27ac peptide were collected at beamline X29 at the National Synchrotron Light Source (NSLS, Brookhaven National Laboratories). All the data sets were integrated and scaled with the program HKL2000 suite. The free-form TRIM24 PHD-Bromo crystal belongs to $P 1$ space group and contains four molecules per asymmetric unit. The structure was solved by the molecular replacement method using the PHASER program ${ }^{51}$, using the BHC80 PHD finger (PDB coordinate 2PUY) and TRIM24 bromodomain (PDB coordinate $2 \mathrm{YYN}$ ) as search models, while searching for four copies of each domain in the asymmetric unit. The initial model was rebuilt with $\mathrm{COOT}^{52}$, cycled with structure refinement by $\mathrm{CNS}^{53}$. The structures of all the complexes were solved by molecular replacement with free-form TRIM24 PHD-Bromo as the model. All the crystallographic statistics are listed in Supplementary Table 1.

Peptide synthesis and fluorescence polarization based measurement. $\mathrm{H} 3$ peptides (residues 1-33) used for isothermal titration calorimetry (ITC) and for fluorescence polarization (FP) measurements were synthesized by FMOC strategy using pseudo prolines at critical positions. Details of peptide synthesis are available on request. For FP assays, peptides were labelled using fluorescein-NHS ester (Invitrogen). Labelled peptides were purified on G10 gel filtration resin (GE Healthcare) and by RP-C18 HPLC. Single labelled species were identified by mass spectrometry. FP assays were essentially carried out and analysed as described using FP buffer $(10 \mathrm{mM} \text { Tris- } \mathrm{HCl}, 100 \mathrm{mM} \mathrm{NaCl}, 5 \mathrm{mM} \text { DTT, pH 7.4 })^{54}$. Titration series of $10 \mu \mathrm{l}$ volume in 384-well plates were read multiple times on a Plate Chameleon II plate reader (HIDEX Oy). Multiple readings and independent titration series were averaged after data normalization.

ITC measurements. Calorimetric experiments were conducted at $25^{\circ} \mathrm{C}$ with a MicroCal iTC200 instrument. Recombinant wild-type TRIM24 PHD-Bromo and its mutants were dialysed overnight against $20 \mathrm{mM}$ Tris ( $\mathrm{pH} 7.5), 50 \mathrm{mM} \mathrm{NaCl}$ and $2 \mathrm{mM} \beta$-mercaptoethanol. Aliquots of lyophilized peptides were dissolved in the same buffer before use. Calorimetric titration was performed by injecting synthetic peptide into wild-type TRIM24 PHD-Bromo or its mutants at various concentrations. Calorimetric titration data were fitted using Origin 7.0 software on the basis of a 1:1 binding stoichiometry.

Peptides used for the crystallization and binding assays. H3(1-10)K4, ARTKQTARKS; H3(1-21)K4, ARTKQTARKSTGGKAPRKQLAGGK-Biotin; H3(1-15)K4, ARTKQTARKSTGGKAY; H3(1-15)K4me1, ARTK(me1)QTARK STGGKAY; H3(1-15)K4me2, ARTK(me2)QTARKSTGGKAY; H3(1-15)K4me3, ARTK(me3)QTARKSTGGKAY; H3(1-19)K14ac, ARTKQTARKSTGGK(ac)A PRKQ; H3(1-20)K9ac, ARTKQTARK(ac)STGGKAPRKQL; H3(23-31)K27ac, KAARK(ac)SAPA; H3(13-32)K27ac, GKAPRKQLATKAARK(ac)SAPATYKBiotin; H3(13-32)K23ac, GKAPRKQLATK(ac)AARKSAPATYK-Biotin; H3(133)K4K23ac-Bio, ARTKQTARKSTGGKAPRKQLATK(ac)AARKSAPATGYKBiotin; H3(1-33)K4me3K23ac-Bio, ARTK(me3)QTARKSTGGKAPRKQLAT K(ac)AARKSAPATGYK-Biotin; H3(1-33)K4-Bio, ARTKQTARKSTGGKAPR KQLATKAARKSAPATGYK-Biotin; H3(1-33)K4, ARTKQTARKSTGGKAPRK QLATKAARKSAPATG; H3K4K23ac, ARTKQTARKSTGGKAPRKQLAT K(ac)AARKSAPATG; H3K4me3K23ac, ARTK(me3)QTARKSTGGKAPRKQL ATK(ac)AARKSAPATG; H4(14-19)K16ac, GAK(ac)RHR; H4(1-20)K16ac, SGRGKGGKGLGKGGAK(ac)RHRK; H4(1-21), SGRGKGGKGLGKGGAKRHR KVGGK-Biotin; H4(1-27)K5ac，SGRGK(ac)GGKGLGKGGAKRHRKVLRDNI Q-PEG-Biotin; H4(1-27)K8ac, SGRGKGGK(ac)GLGKGGAKRHRKVLRDNIQPEG-Biotin; H4(1-27)K12ac，SGRGKGGKGLGK(ac)GGAKRHRKVLRDNIQPEG-Biotin; H4(1-27)K16ac，SGRGKGGKGLGKGGAK(ac)RHRKVLRDNIQPEG-Biotin; H4(1-27)K20ac，SGRGKGGKGLGKGGAKRHRK(ac)VLRDNIQPEG-Biotin.

44. Tsai, F. T. \& Sigler, P. B. Structural basis of preinitiation complex assembly on human pol II promoters. EMBO J. 19, 25-36 (2000).

45. Tsai, W. W., Nguyen, T. T., Shi, Y. \& Barton, M. C. p53-targeted LSD1 functions in repression of chromatin structure and transcription in vivo. Mol. Cell. Biol. 28, 5139-5146 (2008).

46. Bonéy-Montoya, J. et al. Long-range transcriptional control of progesterone receptor gene expression. Mol. Endocrinol. 24, 346-358 (2010).

47. Kuhn, R. M. et al. The UCSC Genome Browser Database: update 2009. Nucleic Acids Res. 37 (Database issue), D755-D761 (2009).

48. Bentley, D. R. et al. Accurate whole human genome sequencing using reversible terminator chemistry. Nature 456, 53-59 (2008).

49. Zhang, Y. et al. Model-based analysis of ChIP-Seq (MACS). Genome Biol. 9, R137 (2008).

50. Shi, X. et al. Proteome-wide analysis in Saccharomyces cerevisiae identifies severa PHD fingers as novel direct and selective binding modules of histone $\mathrm{H3}$ methylated at either lysine 4 or lysine 36. J. Biol. Chem. 282, 2450-2455 (2007)

51. McCoy, A. J. Solving structures of protein complexes by molecular replacement with Phaser. Acta Crystallogr. D. 63, 32-41 (2007).

52. Emsley, P. \& Cowtan, K. Coot: model-building tools for molecular graphics. Acta Crystallogr. D 60, 2126-2132 (2004).

53. Brunger, A.T.Version 1.2 of the Crystallography and NMR system. Nature Protocols 2, 2728-2733 (2007)

54. Jacobs, S. A. Fischle, W. \& Khorasanizadeh, S. Assays for the determination of structure and dynamics of the interaction of the chromodomain with histone peptides. Methods Enzymol. 376, 131-148 (2004). 